\title{
Lesão iatrogênica meningomedular em um cão submetido à espondilectomia ventral para tratamento de extrusão crônica de disco intervertebral cervical
}

\author{
[Iatrogenic meningo-medullary lesion in a dog undergoing ventral spondylectomy for treatment of \\ a chronic cervical intervertebral disc extrusion] \\ B.M. Araújo ${ }^{1}$, M.L. Figueiredo ${ }^{1}$, A.C. Silva ${ }^{1}$, T.H.T. Fernandes ${ }^{1}$, \\ M.A. Bonelli ${ }^{1}$, E.A. Tudury ${ }^{2}$ \\ ${ }^{1}$ Aluno de pós-graduação - Universidade Federal Rural de Pernambuco - Recife, PE \\ ${ }^{2}$ Universidade Federal Rural de Pernambuco - Recife, PE

\section{RESUMO}

Objetivou-se descrever a ocorrência de lesão iatrogênica meningomedular em um cão da raça Dachshund, macho, oito anos de idade, apresentando tetraparesia flácida há dois meses, que foi submetido à espondilectomia ventral para tratamento de extrusão crônica de disco intervertebral cervical. Durante remoção do material de disco herniado com removedores de tártaro e pinça hemostática, observou-se severa hemorragia dos seios venosos, descolamento meníngeo com laceração medular e imediata queda dos parâmetros cardiovasculorrespiratório, com evolução para a morte ante a ausência de respostas às medidas de ressuscitação cardiopulmonar.

Palavras-chave: cão, doença do disco intervertebral, região cervical

\begin{abstract}
Our objective was to describe the occurrence of an iatrogenic meningo-medullary lesion and the transsurgical complications in an eight-year-old male Daschund, which presented flacid tetraparesis for two months, and underwent ventral spondylectomy to treat a chronic cervical intervertebral disc extrusion. During the removal of the herniated disc with dental picks and hemostatic forceps, we observed meningeal dislocation with medullary laceration and an immediate reduction in cardiovasculatory and respiratory parameters, which progressed to a cardiorespiratory arrest that was non-responsive to medication and finally led to the death of the patient.
\end{abstract}

Keywords: dog, intervertebral disk disease, cervical region

\section{INTRODUÇÃO}

A doença do disco intervertebral (DDIV) da região cervical é um frequente distúrbio em cães, correspondendo a aproximadamente $15 \%$ de todas as extrusões de disco intervertebral na espécie canina (Wheller e Sharp, 1999; Cherrone et al., 2004; Seim III, 2008).

Animais de raças de pequeno porte, particularmente aquelas com características condrodistróficas, são mais afetados, mas a doença pode ocorrer em qualquer raça. Oitenta por cento das extrusões de disco cervical ocorrem em Dachshunds, Beagles e Poodles (Wheller e Sharp, 1999; Seim III, 2008).
O sinal clínico predominante é dor cervical grave, frequentemente não responsiva à medicação (Wheller e Sharp, 1999; Lecouteur e Grandy, 2004; Dewey, 2006; Sharp e Wheller, 2006; Seim III, 2008; Santini et al., 2010). Sinais neurológicos relacionados à compressão da medula espinhal cervical ou de raízes nervosas podem ser vistos, sendo a tetraparesia ou a claudicação de um membro torácico os mais frequentes (Wheller e Sharp, 1999).

O tratamento conservativo (repouso absoluto em gaiola e medicação anti-inflamatória) pode ser indicado para a maioria dos pacientes, a menos que estejam presentes marcantes deficiências neurológicas (Wheller e Sharp, 1999).

Recebido em 2 de março de 2012

Aceito em 6 de março de 2013

E-mail: bmaraujo85@hotmail.com 
Animais com dor cervical grave, em geral, não respondem ao tratamento conservativo; costumam ter grande quantidade de material de disco dentro do canal vertebral, e os cães que não apresentam melhora no decorrer de sete a 10 dias de confinamento devem ser avaliados, considerando-se a descompressão cervical (Lecouteur e Grandy, 2004).

O tratamento cirúrgico é a modalidade terapêutica mais indicada para pacientes com DDIV cervical com déficits neurológicos moderados a graves (tetraparesia, tetraplegia) e deve ser realizado sem demora. Pacientes que apresentem tetraplegia aguda devem ser considerados como emergência cirúrgica (Dewey, 2006).

O procedimento clássico, realizado na maioria dos casos, é a espondilectomia ventral, que consiste na abertura de uma janela óssea ventral, envolvendo uma pequena porção do corpo das duas vértebras contíguas ao disco afetado, para obter acesso ao canal vertebral e retirar o material herniado. Porém, se a cirurgia demorar de duas a três semanas, o material de disco endurece e adere à dura-máter, o que dificulta ou impossibilita sua remoção do canal medular (Fry et al.,1991; Padilha Filho e Selmi, 1999; Wheller e Sharp, 1999; Lecouteur e Grandy, 2004).

Objetivou-se descrever a ocorrência de lesão iatrogênica meningomedular e as complicações transcirúrgicas em um caso crônico de DDIV cervical, ressaltando-se a importância do tratamento precoce desse tipo de afecção em consequência da possibilidade de aderência do material do disco à dura-máter, a qual dificulta sua remoção.

\section{CASUÍSTICA}

Foi atendido um cão da raça Dachshund, macho, oito anos de idade, que apresentava tetraparesia flácida há dois meses, após queda de uma altura de dois metros. O animal foi tratado com antiinflamatório e analgésico por outros profissionais durante esse período, sem resolução do caso.

No exame clínico, o animal apresentava todos os parâmetros fisiológicos dentro do padrão para a espécie. No exame neurológico, apresentava tetraparesia flácida simétrica, com ausência de propriocepção e outras reações posturais em todos os membros. A consciência e os reflexos cranianos encontravam-se sem alterações, com reflexos espinhais e cutâneo do tronco presentes e normais, enquanto existiam tensão da musculatura e hiperpatia na região cervical, com sensibilidade superficial e profunda preservada em todos os membros e cauda.

Diante dos dados da resenha, anamnese e dos achados dos exames clínico e neurológico, constatou-se uma síndrome neurológica cervical, provavelmente causada por fratura e/ou luxação vertebral ou por extrusão traumática de disco intervertebral. Foram solicitados exames hematológico e radiográfico (simples e contrastado) da região e prescritos tratamento conservativo com meloxicam $(0,1 \mathrm{mg} / \mathrm{kg}$, SID, por sete dias), cloridrato de tramadol $(1 \mathrm{mg} / \mathrm{kg}$, TID, durante cinco dias) e repouso absoluto em gaiola enquanto eram aguardados os resultados dos exames.

Nos exames físico e hematológico, todos os parâmetros estavam dentro do padrão para a espécie. No exame radiográfico simples, observou-se diminuição do espaço intervertebral entre $\mathrm{C}_{5}-\mathrm{C}_{6}$, sem sinais de fratura ou luxação vertebral (Fig. 1-A). Na mielografia, observou-se elevação da coluna de contraste no espaço intervertebral entre $\mathrm{C}_{5}-\mathrm{C}_{6}$ Fig. 1-B), sendo diagnosticada DDIV cervical.

Diante dos déficits neurológicos graves e da ausência de resposta ao tratamento conservativo pregresso, optou-se pelo tratamento cirúrgico por meio da espondilectomia ventral em sulco para descompressão medular e remoção do material discal de dentro do canal vertebral.

No protocolo anestésico, utilizou-se como medicação pré-anestésica o sulfato de atropina $(0,04 \mathrm{mg} / \mathrm{kg}$, intramuscular), succinato sódico de metilprednisolona $(30 \mathrm{mg} / \mathrm{kg}$, endovenoso (EV) lento, com repetição de $15 \mathrm{mg} / \mathrm{kg}$ duas horas após) e fentanil $(5 \mu \mathrm{g} / \mathrm{kg} \mathrm{EV})$. Para indução anestésica, utilizou-se propofol $(5 \mathrm{mg} / \mathrm{kg} \mathrm{EV})$, e após entubação com sonda rígida, a manutenção se deu pela administração de isoflurano diluído em oxigênio $100 \%$, sendo ainda realizada a administração de fentanil $(2 \mu \mathrm{g} / \mathrm{kg}$ EV) a cada 20 minutos. 


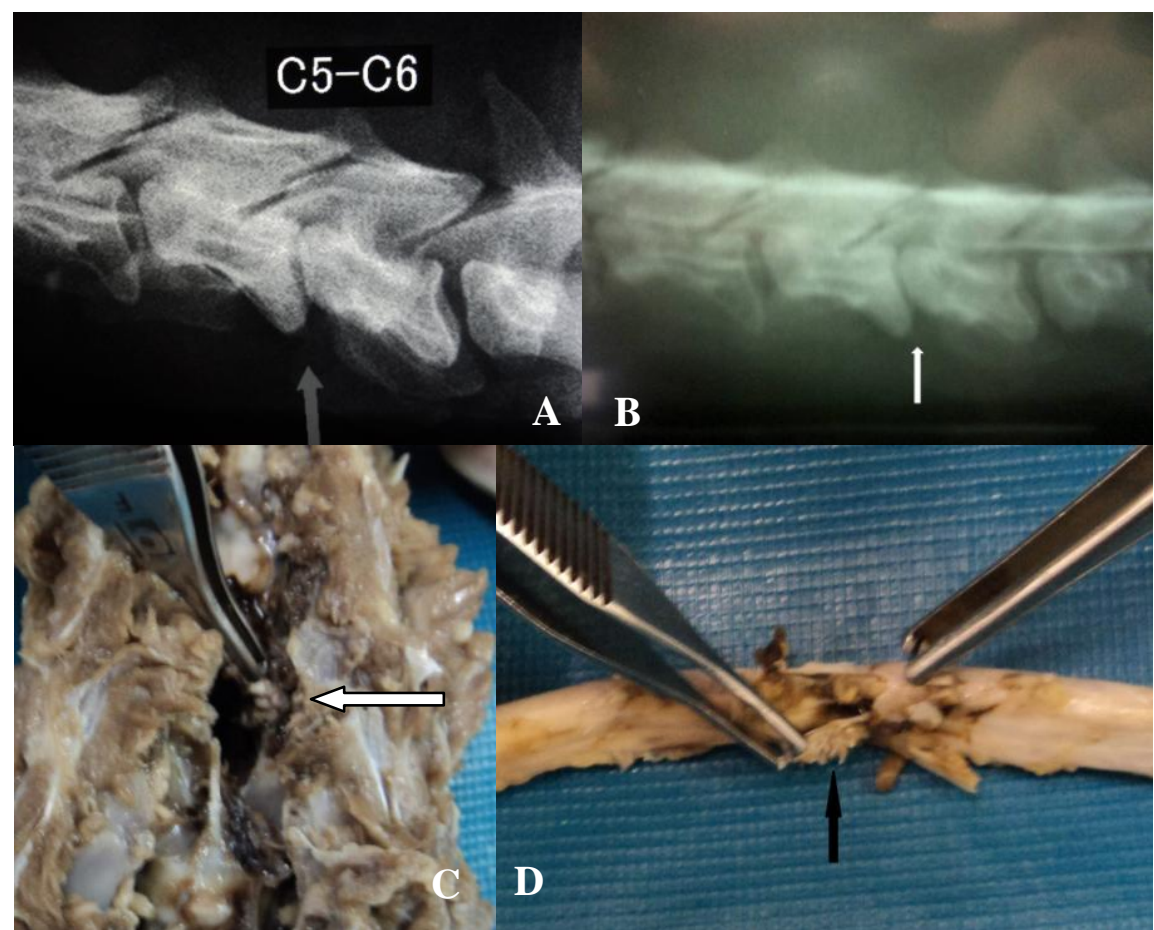

Figura 1. A - Exame radiográfico da coluna cervical, na projeção lateral, demonstrando diminuição de espaço intervertebral entre as vértebras $\mathrm{C}_{5}-\mathrm{C}_{6}$ (seta). $\mathrm{B}-$ Mielografia da coluna cervical, demonstrando elevação da coluna de contraste no espaço intervertebral entre $\mathrm{C}_{5}-\mathrm{C}_{6}$ (seta). $\mathrm{C}-$ Moderada quantidade de material de disco intervertebral no canal vertebral entre $\mathrm{C}_{5}-\mathrm{C}_{6}$ (seta) $\mathrm{D}$ - Material de disco aderido à duramáter (seta), com presença de dilaceração meningomedular (seta).

Para o procedimento cirúrgico, utilizou-se a abordagem descrita por Sharp e Wheller (2006). Após a entrada no canal vertebral, ocorreu severa hemorragia por laceração dos seios venosos, o que dificultou a identificação das estruturas neurais. Após controle parcial da hemorragia por meio da utilização de Surgicell® e Surgiflo® e pinçamento temporário das artérias carótidas com pinças hemostáticas temporárias de Serrefine, identificou-se o material de disco herniado dentro do canal vertebral, que se encontrava bastante aderido à dura-máter. Durante a remoção dele com removedores de tártaro e pinça hemostática, com visibilização prejudicada pela hemorragia local, observou-se descolamento meníngeo com laceração medular e imediata queda dos parâmetros cardiovasculorrespiratório, com evolução para hipotensão e parada cardiorrespiratória não responsiva à ventilação mecânica com oxigênio a $100 \%$, expansão de volume com soluções de ringer com lactato e de cloreto de sódio a 7,5\%, massagem cardíaca e a administração de atropina e catecolaminas, levando o animal a óbito.
$\mathrm{Na}$ necropsia, observou-se, acima do sulco da espondilectomia, a dilaceração meningomedular e dos seios venosos, com grande quantidade de coágulos e resto de moderada quantidade de material de disco intervertebral no canal medular, onde ele se encontrava aderido à duramáter (Fig. 1 - C e D).

\section{DISCUSSÃO}

O diagnóstico de extrusão cervical de disco intervertebral foi obtido por meio dos dados da resenha, da anamnese, do exame clínico geral e neurológico e dos exames de imagem (Lecouteur e Grandy, 2004; Santini et al., 2010).

Diante da dor cervical grave e da tetraparesia, com ausência de resposta ao tratamento conservativo, optou-se pelo tratamento cirúrgico, conforme descrito por Lecouteur e Grandy (2004), corrobando ainda com Dewey (2006), que menciona que o tratamento cirúrgico é a modalidade terapêutica mais indicada para pacientes com discopatia cervical com déficits neurológicos moderados a grave (tetraparesia, 
tetraplegia), devendo ser realizado sem demora. De acordo com Lecouteur e Grandy (2004) e Toombs e Waters (2007), o material de disco exteriorizado inicia uma reação inflamatória extradural que resulta em aderências fibrosas entre a dura-máter e o material de disco extruído.

A lesão meningomedular foi resultante da tentativa de remoção, ante a existência da hemorragia local e da aderência do material do disco à dura-máter, conforme observado no transcirúrgico e na necropsia. Se a cirurgia de DDIV demorar de duas a três semanas, o material de disco endurece e adere à dura-máter, o que dificulta ou impossibilita sua remoção do canal medular (Lecouteur e Grandy, 2004). A remoção do material discal é geralmente direta, mas pode ser difícil se ele estiver aderido à duramáter (dificuldade agravada pelo reduzido espaço da fenda ventral e pela constante hemorragia local). Esse tecido tem que ser removido por dissecação aguçada, que pode lesionar o plexo venoso ou potencialmente mesmo as meninges e a medula espinhal (Wheller e Sharp, 1999).

Clark (1985) e Steffen et al. (2003) mencionam que, no tronco encefálico, estão localizados os centros responsáveis pelas funções cardiopulmonares e que, em cirurgias nessa região, o trauma, a hemorragia e o edema podem se estender ao tronco encefálico, produzindo déficits dessas funções vitais, o que pode levar o paciente a óbito.

Santini et al. (2010) relataram mortalidade de $25 \%(\mathrm{n}=7)$ dos cães com DDIV de seu estudo durante o período transoperatório por bradicardia e hipotensão não responsivas ao tratamento farmacológico. Em outro estudo sobre as causas de mortalidade transoperatória e pós-operatória imediata em cães submetidos à descompressão cervical, Clark (1985) relatou 5\% de óbito (3 de 52) devido a choque hemorrágico e deficiência respiratória. Esse autor atribuiu a mortalidade à natureza radical do procedimento e da manipulação mais extensa da medula espinhal necessária em alguns casos. Em todos os três cães, os danos aos elementos neurais, independentemente da localização, foram suficientes para causar o óbito.

Em relação à hemorragia, esse caso ilustra a possibilidade de consequências indesejáveis na técnica de espondilectomia cervical ventral, por laceração intraoperatória dos seios venosos vertebrais, que estão localizados na face ventral do espaço epidural do canal vertebral.

Apesar da tentativa de uso das bem descritas técnicas de hemostasia, o animal perdeu grande volume de sangue. De acordo com Clark (1985), os cães que sofrem descompressão ventral da coluna vertebral cervical estão em risco de hemorragia grave por danos acidentais aos seios venosos, que se encontram em estreita relação anatômica com o disco intervertebral e as estruturas associadas. As tentativas de hemostasia local são prejudicadas pelo estreito espaço da fenda ventral e, assim como aconteceu no caso relatado por Clark (1985), o sangue para transfusão, que teria sido benéfico neste caso, conforme citado por Denny e Butterworth (2000), não estava disponível imediatamente para transfusão.

\section{CONCLUSÃO}

A extrusão de disco intervertebral é um distúrbio frequente em cães. Desta forma, este trabalho ressalta a importância do encaminhamento precoce dos casos graves a centros especializados, para que o tratamento cirúrgico seja instituído antes do endurecimento e da aderência do material discal à dura-máter, o que diminuiria a possibilidade de complicações trans e pós-cirúrgicas.

\section{REFERÊNCIAS}

CHERRONE, K.L.; DEWEY, C.W.; COATES, J.R.; BERGMAN, R.L. A retrospective comparison of cervical intervertebral disk disease in nonchondrodystrophic large dogs versus small dogs. Am. Anim. Hosp. Assoc., v.40, p.316-320, 2004.

CLARK, D.M. Analysis of intraoperative and early postoperative mortality associated with cervical spinal descompressive surgery in the dog. J. Am. Anim. Hosp. Assoc., v.22, p.739-744, 1986.

DENNY, H.R.; BUTTERWORTH, S.J. 4. ed. A Guide to Canine and Feline Orthopaedic Surgery. Oxford: Blackwell Science Ltd., 2000. $634 \mathrm{p}$. 
DEWEY, W. Neurologia de cães e gatos. Guia prático. São Paulo: Roca, 2006. 352p.

FRY, T.R.; JOHNSON, A.L.; HUNGERFORD, L.; TOOMBS, J. Surgical treatment of cervical disc herniations in ambulatory dogs. Ventral decompression vs. fenestration, 111 cases (19801988). Progr. Vet. Neurol., v.2, p.165-173, 1991.

LECOUTEUR, R.A.; GRANDY, J.L. Doenças da medula espinhal. In: ETTINGER, S. J.; FELDMAN, E.C. 5.ed. Tratado de medicina interna veterinária: Moléstias do cão e do gato. Rio de Janeiro: Guanabara Koogan, 2004. p.66494.

PADILHA FILHO, J.G.; SELMI, A.L. Discopatia cervical no cão. Tratamento cirúrgico através de fenestração ventral. Estudo retrospectivo (1986-1997). Cienc. Rural, v.29, p.75-78, 1999.

SANTINI, G.; MAZZANTI, A.; BECKMANN, D.V. et al. Doença do disco intervertebral cervical em cães: 28 casos (2003-2008). Pesq. Vet. Bras., v.30, p.659-664, 2010.
SEIM III, H.B. Cirurgia da coluna cervical. In: FOSSUM, T.W. 3.ed. Cirurgia de pequenos animais. Rio de Janeiro: Elsevier, 2008. p.14021459.

SHARP, N.J.H.; WHELLER, S.J. 2. ed. Transtornos vertebrales de pequeños animales: diagnóstico y cirurgía. Madrid: Elsevier, 2006. $375 \mathrm{p}$.

STEFFEN, F.; FLUECKIGER, M.; MONTAVON, P.M. Traumatic atlanto-occipital luxation in a dog: associated hypoglossal nerve deficits and use of 3-dimensional computed tomography. Vet. Surg., v.32. p.411-4155, 2003.

TOOMBS, J.P.; WATERS, D.J. Afecção do disco intervertebral. In: SLATTER, D. 3. Ed. Manual de cirurgia de pequenos animais. São Paulo: Manole, 2007. p.1193-1208.

WHELLER, S.J.; SHARP, N.J.H. Diagnóstico e tratamento cirúrgico das afecções espinhais do cão e do gato. São Paulo: Manole, 1999. 224p. 